\title{
Analisis De Los Resultados Del Modelo De Autoevaluacion De Desempeño En Una Empresa De Servicios
}

\author{
Gaspar Eduardo Fuentes Garza, MBA \\ Universidad Popular Autónoma del Estado de Puebla
}

doi: 10.19044/esj.2017.v13n13p128 URL:http://dx.doi.org/10.19044/esj.2017.v13n13p128

\begin{abstract}
The objective of this article is to analyze the performance evaluation of the service area in an organization. There are differences in the results based on the self-evaluation of the performance of its employees and a final evaluation by its leader (direct supervisor). In carrying out this work, the information of more than 3,000 employees was obtained from the Human Resources area. The result of this comparison shows that $62.5 \%$ of the employees had a self-evaluation that does not coincides with the one carried out by their leader. For 37.5\% with coincidence which demands more training for the writing of the objectives, there is a greater participation of the leader in the process of performance management. This is achieved by clearly communicating the objectives of the organization and also reporting how performance management is managed.
\end{abstract}

Keywords: Leader, evaluation, performance

\section{Resumen}

El objetivo de este artículo es analizar la evaluación del desempeño en una organización del área de servicios en la que se tiene una autoevaluación del desempeño de sus empleados y una evaluación final por parte de su líder (jefe directo) habiendo aquí diferencias en los resultados. Para realizar este trabajo se obtuvo del área de Recursos Humanos la información de más de 3,000 empleados. El resultado de esta comparación muestra que el 62.5\% de los empleados tuvieron una una autoevaluación que no coincide con la realizada por su líder y un 37.5\% con coincidencia, lo cual demanda una mayor capacitación para la redacción de los objetivos, una mayor participación del líder en el proceso de gestión del desempeño transmitiendo con claridad los objetivos de la organización y también el informar como se administra la gestión del desempeño. 
Palabras clave: Líder, evaluación, desempeño

\section{Introducción}

Toda organización busca cumplir con una visión que la conduzca a un nivel superior respecto al estado actual en el que se encuentra. Dicha visión es la pauta que determina las acciones a realizar por la institución a corto, mediano y largo plazo. Una vez definida la visión y acciones para lograrla, es necesario que todas las personas que forman parte de la organización definan sus objetivos de desempeño, de tal manera que al conseguirlos, impulsen a la organización al cumplimiento de sus metas. El proceso de definición y evaluación de los objetivos son claves para determinar si el recurso humano de la organización está alineado a la visión y metas que la institución se ha establecido. La correcta planificación de metas así como una definición clara, realista y alineada de los objetivos de desempeño, permitirá a todos sus integrantes recibir una evaluación justa por parte de los lideres, al momento de medir el cumplimiento de sus actividades en el tiempo establecido, y de esta forma definir su plan de vida y crecimiento dentro de la institución.En esta investigación se analizará el proceso de evaluación de desempeño de una organización y el impacto negativos que puede haber al no contar con una capacitación adecuada no solo para la definición de objetivos, sino también para la autoevaluación y calibración de los mismos.

\section{Marco Teórico}

Los teóricos de la evaluación difieren ampliamente en la manera de entender qué es y como evaluar. Muchos han implementado con mayor o menor facilidad un modelo conceptual, filosófico e ideológico. Durante las últimas dos décadas se han elaborado alrededor de 50 modelos diferentes de evaluación. La proliferación de modelos es una señal de crecimiento y muestra la complejidad de los problemas metodológicos y conceptuales que pueden encontrarse en la evaluación. Este fenómeno se explica desde el punto de vista filosófico si se tiene en cuenta que evaluar es un proceso subjetivo que refleja la realidad objetiva y cada pensador le imprime el sello de la sociedad y el sistema de valores que le ha tocado vivir, mediante un proceso valorativo. Con la aparición en 1970 del paradigma cualitativo en la investigación educativa y en coincidencia con la evaluación de programas, se comienza a incorporar modelos que se centran en los participantes, es decir se orientan a proporcionar información del proceso de implementación del programa con el objetivo de que los participantes tengan una mayor y más completa información del proceso, lo cual llevará a sus propias conclusiones (Benitez, 2008). 
Chiavenato (2007) sostiene que la evaluación del desempeño es una apreciación sistemática de cómo cada persona se desenvuelve en un puesto y su potencial de desarrollo futuro, lo anterior con el fin de:

1. Garantizar un clima laboral de confianza y respeto entre las personas.

2. $\quad$ Propiciar que las personas asuman responsabilidades y definan metas de trabajo.

3. Desarrollar un estilo de administración democrático, participativo y consultivo.

4. Crear un propósito de dirección, futuro y mejora continua de las personas.

5. Generar una expectativa permanente de aprendizaje, innovación, desarrollo personal y profesional.

En comparacion con lo expresado por Chiavenato, la Asociasion Española para la Calidad (2016) da a concoer que la evaluación de desempeño es un proceso sistemático y periódico de estimación cuantitativa y cualitativa del grado de eficacia con el que las personas llevan a cabo las actividades y responsabilidades de los puestos que desarrollan. Su finalidad es juzgar o estimar el valor, la existencia y las cualidades de una persona y, sobre todo, su contribución a la organización. La Asocición tambien da a conocer que la Organización Internacional ISO, ha publicado en 2012 la norma ISO 10667: 2012. Procedimientos y métodos para la evalución de personas en entornos laborales y contextos organizacionales, esta norma tiene como misión proporcionar una guía clara y consisa para las buenas prácticas en evaluación de personas en contextos laborales, y lo hace desde una perspectiva basada en evidencia, medible y aplicable a nivel mundial. En la siguiente tabla se detallan algunos ejemplos de modelos de evaluación del desempeño expuestos por Mejía (2001):

Tabla 1. Métodos de evaluación del desempeño

\begin{tabular}{|c|c|c|c|}
\hline Evaluación $360^{\circ}$ & $\begin{array}{l}\text { Es realizada por } \\
\text { todos los elementos } \\
\text { que tienen algún } \\
\text { tipo de interacción } \\
\text { con el evaluado }\end{array}$ & $\begin{array}{c}\text { Evaluaciones desde } \\
\text { muchas perspectivas. } \\
\text { La variedad de } \\
\text { opiniones ayuda a } \\
\text { enriquecer la función } \\
\text { del evaluado. }\end{array}$ & $\begin{array}{l}\text { Es un sistema } \\
\text { complejo. } \\
\text { Puede afectar el } \\
\text { clima y las } \\
\text { relaciones. }\end{array}$ \\
\hline Escalas gráficas & $\begin{array}{c}\text { Es el más empleado } \\
\text { y divulgado y } \\
\text { simple. } \\
\text { Usa factores } \\
\text { previamente } \\
\text { definidos. }\end{array}$ & $\begin{array}{l}\text { Instrumento fácil de } \\
\text { entender y aplicar. } \\
\text { Permite una visión } \\
\text { integral y resumida. }\end{array}$ & $\begin{array}{l}\text { No brinda } \\
\text { flexibilidad al } \\
\text { evaluador. } \\
\text { Tiende a ser } \\
\text { rutinario. }\end{array}$ \\
\hline Elección forzosa & $\begin{array}{l}\text { El evaluador está } \\
\text { obligado a escoger } \\
\text { una o dos frases de }\end{array}$ & $\begin{array}{c}\text { Proporciona } \\
\text { resultados confiables } \\
\text { y excentos de }\end{array}$ & $\begin{array}{l}\text { Elaboración } \\
\text { compleja. } \\
\text { Es un método }\end{array}$ \\
\hline
\end{tabular}




\begin{tabular}{cccc}
\hline & $\begin{array}{c}\text { un bloque de cuatro } \\
\text { o más frases que } \\
\text { expliquen mejor el } \\
\text { desempeño del } \\
\text { evaluado. }\end{array}$ & $\begin{array}{c}\text { influencias. } \\
\text { Aplicación simple. }\end{array}$ & $\begin{array}{c}\text { comparativo y limita } \\
\text { al evaluado en tres } \\
\text { criterios. }\end{array}$ \\
$\begin{array}{c}\text { Investigación de } \\
\text { campo }\end{array}$ & $\begin{array}{c}\text { Entrevistas hechas } \\
\text { al líder y } \\
\text { subordinados del } \\
\text { evaluado. }\end{array}$ & $\begin{array}{c}\text { Análisis profundo. } \\
\text { Amplitud de } \\
\text { información. }\end{array}$ & $\begin{array}{c}\text { Alto nivel de } \\
\text { complejidad } \\
\text { Sujeta a prejuicios. }\end{array}$ \\
\hline
\end{tabular}

Los colaboradores deben contribuir con el logro de los objetivos de la organización donde se desempeñan, ya que de no hacerlo la empresa desaparecerá tarde ó temprano (Alvarez, 2015). El jefe ó líder jugará un papel primordial tanto para capacitar a sus subordinados en el diseño de sus objetivos así como para servir de modelo invitando al crecimiento y a tener una mejor calidad de vida. Este líder crea un clima de sinergia que facilita el desarrollo de sus colaboradores y los invita a la acción. afirman también, que con esta relación positiva, se refuerza la motivación intrínseca, creatividad y compromiso hacia los resultados, satisfacción, productividad y lealtad (Reynoso \& Lagos, 2015).

El proceso de gestión del desempeño consta de tres etapas: La planeación del desempeño, el seguimiento y acompañamiento y la evaluación para el desarrollo (Montoya, 2009). Cada uno de estos pasos son importantes ya que representan una responsabilidad compartida tanto de los líderes como de sus subordinados para que entre ambos puedan cumplir con la organización y al mismo tiempo generar el desarrollo y crecimiento del individuo en su vida profesional y laboral. En términos concretos, el principal desafío del especialista en recursos humanos es lograr el mejoramiento permanente de las organización de la cual forme parte, haciéndola más eficiente y más eficaz. Ser eficiente implica utilizar la cantidad mínima de recursos necesarios para la producción de bienes y servicios. Ser eficaz implica lograr la producción de estos bienes y servicios adecuados, de manera que sean aceptables para la sociedad (Parra, 2000). La correcta gestión del recurso humano podrá llevar a lograr dicho objetivo.

\section{Descripción del modelo de gestión de desempeño en la empresa sujeto de estudio.}

La institución donde se realizó este estudio es del área de servicios y cuenta con un modelo de evaluación de desempeño implementado hace tres años y opera de la siguiente manera:

- $\quad$ El colaborador establece sus objetivos anuales, los cuales se clasifican de la siguiente manera:

o Objetivos de desempeño 
- $\quad$ Relacionados con las responsabilidades básicas del puesto

o Objetivos de desarrollo

- Actividades específicas para mejorar habilidades, conocimientos y comportamientos relacionados a la actividad laboral.

o Objetivos de transformación y cambio

- Planes específicos para mejorar los métodos de trabajo y clima organizacional.

o Objetivos de desarrollo de mis colaboradores

- Estrategias para apoyar el desarrollo de los colaboradores.

- $\quad$ El colaborador revisa con su líder los objetivos para su aprobación.

- $\quad$ Se hace una revisión intermedia a la mitad del período en la cual el colaborador recibe retroalimentación por parte de su líder y si es necesario se acuerdan ajustes a los objetivos y metas.

- $\quad$ Al finalizar el período correspondiente (1 año) el colaborador realiza una autoevaluación de su desempeño la cual es enviada al líder para su aprobación.

- $\quad$ La escala de evaluación para medir el desempeño es de 1 a 5, donde:

o 5 = Excede lo establecido y está muy por encima de las funciones encomendadas.

o 4 = Cumple por encima de lo establecido y da un valor agregado a su función.

o $\quad 3$ = Cumple plenamente con los compromisos establecidos.

o 2 = Cumple parcialmente con los compromisos establecidos

o $\quad 1=$ No cumple con sus objetivos.

- $\quad$ El líder tiene con sus pares una sesión de calibración en la cual se determina si la autoevaluación coincide con la percepción de quienes tienen una visión de su desempeño y se determina la evaluación correspondiente.

- El líder tiene una sesión de informe de desempeño con su coloborador.

- $\quad$ Si el colaborador tiene una evaluación de cuatro (4) ó (5) recibe una compensación económica y también se empieza a trabajar en su plan de crecimiento y desarrollo.

- $\quad$ Si el colabordor tiene un tres (3) de evaluación recibe también la prima correspondiente y se le pide un plan de trabajo para llevar ese tres a un cuatro (4) ó cinco (5).

- $\quad$ Si el colaborador tiene una evaluación de dos (2) se hace un plan de mejora el cual es un compromiso del líder y el colaborador para llevarlo a un cumplimiento satisfactorio de objetivos. Una persona con dos evaluaciones igual a dos (2) es candidata a ser reubicada ó desvinculada.

- Un colaborador con evaluación de uno (1) es candidato a ser reubicado ó desvinculado en forma directa. 
En este modelo, cada individuo debe cumplir con su rol y responsabilidades para que de esta forma se le pueda dar un seguimiento puntual y adecuado a los avances en el cumplimiento de los objetivos y que al mismo tiempo garantice que la organización va en dirección correcta. Otro punto importante para la adecuada operación de este modelo, es el contar con un sistema de información que permita desde la captura, la evaluación y el manejo histórico de la información para la toma estratégica de decisiones.

\section{Metodología}

Esta investigación busca comprobar la siguiente hipótesis: $L a$ organización tiene un proceso de evaluación claro para el empleado que en ella labora el cual le permita determinar claramente su nivel de desempeño. Teniendo como variables la autoevaluación y la evaluación en un período de tiempo determinado.

El proceso de evaluación se realizó con una metodología descriptiva que ayudó a detallar cómo se presenta una situación determinada. Se emplearón dos tipos de investigación, por un lado se adoptó la cuantitativa para analizar los resultados de la autoevaluación y evaluación final de los colaboradores. Por otro lado se empleó la investigación cualitativa que implicó recolectar la opinión de los líderes de primer nivel de la organización respecto a los resultados generados.

La evaluación cuantitativa implicó solicitar al área de Recursos Humanos de la organización un listado que contiene por cada empleado la autoevaluación realizada por éste y la evaluación final que dio su jefe directo. Con esta información se hizo la revisión y se determinó el número de coincidencias.

La evaluación cualitativa se hizo a través de una consulta a los líderes de la organización a quienes se les presentaron las coincidencias y diferencias resultantes del estudio cuantitativo para conocer su opinión sobre el porqué consideran que se dan dichos resultados. Dicha información se expresó por escrito.

\section{Detalle de la Implementación}

- $\quad$ La población de la organización cuyos niveles jerárquicos ocupan generar objetivos es de 3378 individuos. De todos ellos se obtuvieron sus autoevaluaciones y sus evaluaciones finales las cuales son el resultado de la calibración hecha por sus líderes.

- $\quad$ La información de la autoevaluación así como de la evaluación final fue proporcionada por la Dirección de Recursos Humanos de la organización quien también, para la segunda fase del estudio, ayudó a contactar a todos los miembros del equipo directivo de primer nivel jerárquico el cual consta de un 
grupo de 17 líderes y al equipo del segundo nivel de jerarquía el cual consta de un total de 136 colaboradores.

- $\quad$ A cada uno de los líderes se les solicitó generar un listado donde mencionaran a su juicio las cinco posibles causas que generan este comportamiento obtenido en el análisis de la información de las evaluaciones.

\section{Resultados}

Después de hacer las comparaciones de la autoevaluación hecha por los colaboradores y la evaluación final realizada por su líder se generó la información correspondiente la cual se presenta en la tabla 2.

Tabla 2. Resultados de la autoevaluación y evaluación final recibida por los colaboradores

\begin{tabular}{|c|c|c|c|c|c|c|}
\cline { 2 - 7 } \multicolumn{2}{c|}{} & \multicolumn{5}{c|}{ EVALUACION } \\
\cline { 2 - 7 } & 5 & 4 & 3 & 2 & 1 \\
\hline \multirow{2}{*}{$\frac{5}{3}$} & 5 & 18 & 56 & 74 & 29 & 6 \\
\cline { 2 - 7 } & 4 & 34 & 186 & 407 & 229 & 25 \\
\cline { 2 - 7 } & 3 & 37 & 266 & 983 & 713 & 100 \\
\cline { 2 - 7 } & 2 & 7 & 16 & 75 & 81 & 29 \\
\hline
\end{tabular}

En esta tabla se puede observar que:

- $\quad$ 1,668 personas las cuales representan un $49.4 \%$ de la población tuvieron una evaluación final menor a la autoevaluada.

- $\quad 442$ personas las cuales representan el $13.1 \%$ de la población tuvieron una evaluación final superior a la autoevaluada.

- $\quad \mathbf{1 , 2 6 8}$ personas las cuales representan el $\mathbf{3 7 . 5 \%}$ de la población tuvieron coincidencias entre su autoevaluación y la evaluación final.

Es importante hacer mención que $\mathbf{2 , 1 1 0}$ personas las cuales representan el $\mathbf{6 2 . 5 \%}$ de la población no se autoevaluaron de manera correcta, es decir se evaluaron mejor sabiendo que su desempeño no fue el esperado o bien se evaluaron mal pero al parecer de sus líderes, su desempeño fue satisfactorio.

Esta información se compartió en formato electrónico a los 153 líderes de primer y segundo nivel jerárquico para que compartieran 5 opiniones que consideran fueran las que generan esta situación. 
Cada opinión fue filtrada para ser agrupada en una categoría y se dan a conocer en la tabla 3 en la cual el factor más mencionado es la mala redacción de los objetivos por parte de los colaboradores, el segundo factor es que el líder no transmite las metas del área en forma adecuada y el tercero es que el modelo aún no ha sido bien adoptado por la organización.

Tabla 3. Principales causas de error en la autoevaluación del desempeño

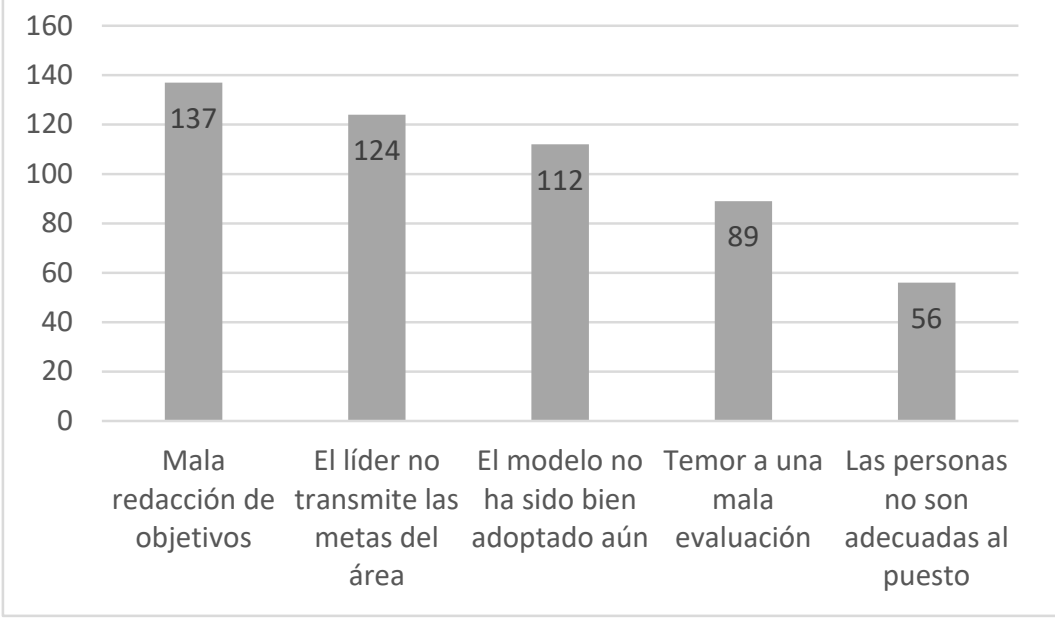

\section{Conclusion}

De acuerdo al análisis de la información presentada se puede concluir que a tres años de haberse implementado este modelo de gestión del desempeño, más de la mitad de la población que compone la organización aún no hace una autoevaluación de su desempeño de forma correcta.

También se rechaza la hípotesis al demostrarse que aún no se cuenta con un modelo de evaluación del desempeño claro que permita al empleado evaluarse de forma objetiva.

Se deduce que las principales causas de error en la autoevaluación de desempeño, se debe a:

- A una mala redacción de objetivos.

- $\quad$ A que el líder no trasmite de forma adecuada las metas de su área.

- $\quad$ El modelo de gestión de desempeño no se ha adoptado bien por la organización.

- $\quad$ Hay un temor a una mala evaluación por parte del líder y por eso se autoevaluán por encima de lo real.

- $\quad$ Que las personas no están calificadas para los puestos en el que están desempeñándose.

El líder es la clave para corregir esta situación, ya que debe trabajar con los integrantes de su equipo trasmitiéndoles hacia dónde va la dirección del departamento y de la organización, de igual manera debe asesorarlos en el 
establecimiento y redacción de sus objetivos de desempeño de tal manera que éstos sean claros, medibles y alcanzables.

El llevar a cabo un proceso de evaluación del desempeño claro, confiable y objetivo es clave para el buen funcionamiento de toda organización, por el contrario, de no ser así puede afectar el futuro no solo de la organización, sino también de su recurso más valioso que es el capital humano que la conforma.

\section{References:}

1. Alvarez, W. (2015). La evaluación del desempeño basada en competencias. Conexion ESAN. Lima, Perú: ESAN Perú.

2. Asociacion Española Para la Calidad (2016). Evaluación de Desempeño. Madrid. Recuperado de http://www.aec.es/web/guest/centro-conocimiento/evaluacion-dedesempeno

3. Benítez, M., Martínez, M., \& Rubio, J. (2008). Propuesta de un programa de evaluación de desempeño basado en el desarrollo de competencias para mejorar la efectividad laboral del Hotel Trópico Inn en la Ciudad de San Miguel. Trabajo de graduación, El Salvador. Recuperado http://www.univo.edu.sv:8081/tesis/019076/019076_Cap2.pdf

4. Chiavenato, I. (2007). Administración de los Recursos Humanos 8a Ed. México DF, México: McGraw-Hill.

5. Mejía, L. (2001). Dirección y Gestión de Recursos Humanos. Madrid, EspañaP: Pearson Eudcation.

6. Montoya, C. (2009). Evaluación del Desempeño como Herramienta para el Análisis del Capital Humano. Recuperado de: http://www.scielo.org.ar/scielo.php?script=sci_arttext\&pid=S166887082009000100002\&lng=es\&nrm=iso

7. Parra, M. (2000). La evaluación del desempeño y la gestión de RRHH. Recuperado de http://www.rrhhmagazine.com/articulos.asp?id=28

8. Reynoso, C., \& Lagos, C. (2015). Socialización y Engagement, Global Conference on Business and Finance. Business Source Elite, 10, 778-787.

9. Sampieri, R, Fernández, C, \& Baptista, P. (2010). Metodología de la investigación (5ta. ed.). D.F., México: McGraw Hill. 